\title{
Edge logarithmic corrections probed by impurity NMR
}

\author{
V. Brunel, M. Bocquet and Th. Jolicœur* \\ Service de Physique Théorique, CEA Saclay, F91191 Gif-sur-Yvette, France
}

(April 27, 2018)

\begin{abstract}
Semi-infinite quantum spin chains display spin autocorrelations near the boundary with power-law exponents that are given by boundary conformal field theories. We show that NMR measurements on spinless impurities that break a quantum spin chain lead to a spin-lattice relaxation rate $1 / T_{1}^{\text {edge }}$ that has a temperature dependence which is a direct probe of the anomalous boundary exponents. For the antiferromagnetic $\mathrm{S}=1 / 2$ spin chain, we show that $1 / T_{1}^{e d g e} \propto T \log ^{2} T$ instead $\operatorname{of} \log ^{1 / 2} T$ for a bulk measurement. We show that, in the case of a one-dimensional conductor described by a Luttinger liquid, a similar measurement leads to a relaxation rate $1 / T_{1}^{e d g e} \propto T$, independent of the anomalous exponent $K_{\rho}$.
\end{abstract}

75.10.Jm, 75.40.Gb

Impurity effects in quantum spin chains are a subject of much interest since they are a sensitive probe of the peculiar correlations that develop in these magnetic systems. For example it has been shown recently [1] that an external uniform magnetic field creates a staggered magnetization near impurities in the antiferromagnetic (AF) spin chain compound $\mathrm{Sr}_{2} \mathrm{CuO}_{3}$, in agreement with theoretical predictions [2]. In $\mathrm{S}=1 \mathrm{AF}$ spin systems with a Haldane gap in the bulk there are effective free spins $\mathrm{S}=1 / 2$ at the end [3] of the chain that are revealed by the coupling to an impurity.

One-dimensional quantum systems like the $\mathrm{S}=1 / 2 \mathrm{AF}$ chain or the electron gas - the "Luttinger liquid" are critical systems with an effective low-energy physics which is a conformal field theory. In both cases it is the massless Gaussian model that is at the heart of the correct description. When boundaries are introduced in such problems, then it has been shown by Cardy that there are boundary operators with nontrivial scaling dimensions [ 4 . This means that if we measure the spin autocorrelation in time $\langle\mathbf{S}(t) \cdot \mathbf{S}(0)\rangle$ for the spin at the end of a chain, it decays with a power law which is different from that of the bulk. Measurements with a local probe like the $1 / T_{1}$ relaxation rate in NMR experiments are sensitive to this power law.

Conventional NMR is performed on nuclear spins that are coupled by hyperfine interactions to the atomic spin of the same atom : for example in the $\mathrm{S}=1 / 2$ antiferromagnetic spin chain $\mathrm{Sr}_{2} \mathrm{CuO}_{3}$ one may use ${ }^{63} \mathrm{Cu}$ resonance [1]. While this is a local measurement, it involves the time autocorrelation of a bulk spin. We show in this Letter that by NMR measurements on nuclei belonging to spinless impurities that break the chain (or the 1D conductor) one measures the anomalous boundary exponents of the boundary conformal field theory. We call this "edge NMR". In the case of the spin- $1 / 2$ chain we show that the bulk marginal operator manifests itself by a logarithmic factor in $1 / T_{1}$ which is different from the bulk case.

In the case of a spin chain, our main idea is illustrated in fig. 1. Impurities that are spinless but do have a nuclear spin are introduced at a low concentration level in the spin chain. The nuclear spin of the impurity in fig. 1 has substantial coupling only with its two neighboring spins if we are dealing with a magnetic insulator. The coupling may be dipolar or transferred hyperfine : its precise nature does not matter for our reasoning. When impurities are very dilute, the nuclear spin is surrounded by two essentially semi-infinite spin chains. So measurement of the relaxation rate $T_{1}$ of the impurity nuclear spin involve only the fluctuations of the two end spins and these spins give the same contribution. If there is no exchange through the impurity, then we have exactly a probe of a boundary critical phenomena since the relaxation rate is given by the following formula [5] :

$$
1 / T_{1}=A^{2} \lim _{\omega \rightarrow 0} \int_{-\infty}^{+\infty} d t e^{i \omega t}\left\langle\mathbf{S}_{0}(t) \cdot \mathbf{S}_{0}(0)\right\rangle_{T}
$$

In this equation $A$ is the (hyperfine) coupling, $\mathbf{S}_{0}$ is the end spin of the semi-infinite chain and $\langle\ldots\rangle_{T}$ stands for thermal averaging. An example would be a $S=1 / 2$ spin chain realized by $\mathrm{Cu}^{2+}$ ions and the impurity may be $\mathrm{Zn}$ which is a possible substitution for $\mathrm{Cu}$ in the cuprate family of materials. So Zn doping effectively cuts the chains. The isotope ${ }^{67} \mathrm{Zn}$ has a nuclear spin so NMR may be performed on this nucleus selectively.

In the case of the $\mathrm{S}=1 / 2 \mathrm{AF}$ chain we show that $\left(1 / T_{1}\right)^{e d g e} \propto T \log ^{2} T$ is the universal low-temperature behavior instead of $\log ^{1 / 2} T$ as predicted [6, R] and measured in the case of bulk NMR [8]. In the case of a onedimensional conductor, one can perform the same kind of measurement by using an impurity which is insulating, i.e. which breaks the chain. Similarly one has now a relaxation rate that scales as $\left(1 / T_{1}\right)^{\text {edge }} \propto T$ while in the bulk it is given by [9] $\left(1 / T_{1}\right)^{b u l k} \propto T+T^{K_{\rho}}$ where $K_{\rho}$ is the anomalous exponent that characterizes the Luttinger liquid behavior of the system.

We first discuss the $\mathrm{S}=1 / 2 \mathrm{AF}$ spin chain described by the Heisenberg hamiltonian : 


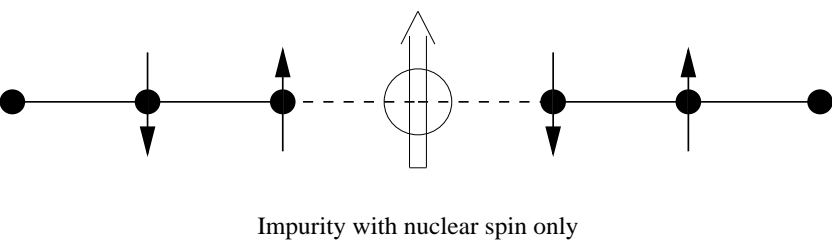

FIG. 1. An NMR $1 / T_{1}$ measurement performed on an impurity with a nuclear spin but no atomic spin (white dot) is a probe of the time autocorrelation of the end spins of the spin chain (black dots).

$$
\mathcal{H}=J \sum_{n} S_{n}^{x} S_{n+1}^{x}+S_{n}^{y} S_{n+1}^{y}+\Delta S_{n}^{z} S_{n+1}^{z}
$$

In this equation, we have introduced the exchange anisotropy $\Delta$ which is convenient for the bosonization description of the low-energy physics. In most materials that are known to be reasonably well described by a $\mathrm{S}=1 / 2 \mathrm{AF}$ chain like $\mathrm{KCuF}_{3}$ or $\mathrm{Sr}_{2} \mathrm{CuO}_{3}$, in fact there is isotropy, i. e. $\Delta=1$. If $\Delta=0$ we have the $\mathrm{XY}$ chain which maps onto a system of free spinless fermions in a band of dispersion $E(k)=-J \cos k$ and two Fermi points at $k_{F}= \pm \pi / 2$. There are particle-hole excitations with small momentum transfer, i.e. staying on the same side of the Fermi "surface" and also excitations across the Fermi surface with momentum transfer $2 k_{F}=\pi$. In the spin language, they correspond respectively to ferro and antiferromagnetic fluctuations. In the bosonic language [10] we have :

$$
S_{x}^{z}=-\frac{1}{\sqrt{\pi}} \nabla_{x} \phi(x)+\frac{(-)^{x}}{\pi \alpha} \cos (\sqrt{4 \pi} \phi(x)) .
$$

The effective theory in the infrared limit for the boson $\phi$ is a free theory given by the following Lagrangian density :

$$
\mathcal{L}_{\phi}=\frac{1}{2}\left[\left(\partial_{t} \phi\right)^{2}-\left(\partial_{x} \phi\right)^{2}\right]
$$

For anisotropies $\Delta \leq 1$, the chain is in a massless phase which is still described by Eq.(1) after a rescaling of the boson $\phi \rightarrow \phi / \sqrt{K}$ where $K=K(\Delta)$ is a parameter that contains the effects of interactions induced by z-exchange. At the isotropic point $\Delta=1$ we have $K=1 / 2$.

This massless effective theory leads to power laws in the spin fluctuations. The local correlations of a spin $\mathbf{S}$ in an infinite chain can be computed at nonzero temperature $T$ with the result :

$$
\begin{gathered}
\left\langle S^{z}(t) S^{z}(0)\right\rangle \propto \frac{T^{2}}{\sinh ^{2} \pi T t}+\left(\frac{T^{2}}{\sinh ^{2} \pi T t}\right)^{K}, \\
\left\langle S^{+}(t) S^{-}(0)\right\rangle \propto\left(\frac{T^{2}}{\sinh ^{2} \pi T t}\right)^{1 / 4 K}+\left(\frac{T}{\sinh \pi T t}\right)^{2 K+1 / 2 K},
\end{gathered}
$$

(omitting constant prefactors). In the isotropic case both spin correlations are equal to $T / \sinh \pi T t$ and this behavior leads to a relaxation $1 / T_{1}$ calculated from Eq. (1) which is $\propto T^{0}$. This is essentially the behavior observed by Takigawa et al. $[8]$. There is an additional logarithmic correction $\log ^{1 / 2} T$ due to the presence of Umklapp processes for the fermions [6].

If we now consider a spin at the end of an open chain, we must supply our bosonic description with a boundary condition. It is a Dirichlet condition which is appropriate [11]. The condition $\left\langle S^{z}(x=0)\right\rangle=0$ in the bosonic theory leads to $\phi(0)=\sqrt{\pi} / 2 \sqrt{2}$ by use of Eq. (3). This boundary condition can then be used to relate the chiral components $\phi_{L}$ and $\phi_{R}$. Indeed $\phi_{R}$ may be regarded as the analytic continuation of $\phi_{L}$ on the negative axis by $\phi_{R}(x, t)=-\phi_{L}(-x, t)+\sqrt{\pi} / 2 \sqrt{2}$. The Green's function can then be expressed in terms of the L field only and this field does not feel the existence of the boundary.

The edge autocorrelations have now a different behavior from the bulk. We find that the uniform and staggered part of the correlation functions have the same behavior at the edge, given by :

$$
\begin{gathered}
\left\langle S^{z}(t) S^{z}(0)\right\rangle \propto \frac{T^{2}}{\sinh ^{2} \pi T t} \\
\left\langle S^{+}(t) S^{-}(0)\right\rangle \propto\left(\frac{T}{\sinh \pi T t}\right)^{1 / K},
\end{gathered}
$$

where we have kept the leading behavior. This is due to the fact that spin operators have boundary scaling dimensions 1 instead of $1 / 2$ in the bulk. In the isotropic case these correlations leads to contributions to $1 / T_{1}$ that scale as $T$ instead of $T^{0}$ in the bulk. So the boundary critical behavior influences directly the NMR relaxation rate. The leading behavior is thus $\left(1 / T_{1}\right)^{e d g e} \propto T$.

We now turn to the influence of the marginal operator. Indeed it is known that there are logarithmic corrections to the power-law behavior of the spin- $1 / 2$ chain 12, 13. In fact the effective theory given by Eq. (14) contains an additional marginal operator that is allowed in the isotropic case : it is due to Umklapp scattering of the fermions and can be written as $\cos (\sqrt{16 \pi} \phi)$. The complete effective theory is given by :

$$
\mathcal{L}_{\phi}=\frac{1}{2}\left[\left(\partial_{t} \phi\right)^{2}-\left(\partial_{x} \phi\right)^{2}\right]+g \cos (\sqrt{16 \pi} \phi) .
$$

The set of the two coupling constants $K$ and $g$ flows under renormalization group according to the KosterlitzThouless equations. There is a single line that reaches the fixed point $K^{*}=1 / 2$ and which corresponds to an isotropic spin chain. Along this line, the flow of the couplings is marginal. This operator leads to a multiplicative logarithmic correction to the bulk magnetic susceptibility, as predicted theoretically [14] and observed experimentally [15]. In the relaxation rate it leads to a correction [6] $\propto(\log T)^{1 / 2}$ which is a possible explanation for 
the experimental upturn of $1 / T_{1}$ at low temperature [8]. Many numerical studies have been devoted to demonstrate $a b$ initio its existence in the spin- $1 / 2$ chain. In fact it is responsible for the extremely slow approach to the thermodynamic limit 16,17.

This bulk marginal operator still exists in the theory with a boundary. In addition, some new operators are permitted at the edge. Note that the surface term $\partial_{x} \phi_{L}$, while forbidden in the bulk of the chain, is a perfectly allowed operator in the boundary theory. If we now classify all possible marginal operators at the boundary fixed point, there are only two such operators $\partial_{x} \phi_{L}$ and $\exp \left(i \sqrt{8 \pi} \phi_{L}\right)$. They have boundary scale dimension 1 and thus are marginal on the edge.

But it is easy to see that they are forbidden by the symmetry of the problem. The rotational invariance around z-axis forbids $\exp \left(i \sqrt{8 \pi} \phi_{L}\right)$ whereas it is rotation around $\mathrm{x}$-axis that forbids $\partial_{x} \phi_{L}$. As a consequence, there are no extra marginal operators. Thus the Green's function defined by :

$$
G(t)^{e d g e}=\left\langle S_{0}^{+}(t) S_{0}^{-}(0)\right\rangle
$$

obeys the following edge renormalization group equation :

$$
\left[t \frac{\partial}{\partial t}+\beta_{b u l k}(g) \frac{\partial}{\partial g}+2 \gamma_{e d g e}(g)\right] G(t, g)^{e d g e}=0
$$

In this equation, $g$ is the coupling of the marginal operator. Since it is a bulk operator its $\beta$-function is that of the bulk : $\beta(g) \equiv \beta_{\text {bulk }}(g)$. The anomalous dimension $\gamma_{\text {edge }}(g)$ however is related to the short-distance singularity of the bulk marginal operator with an operator that lies at the boundary. Hence it is modified with respect to its bulk value.

We compute $\gamma_{\text {edge }}(g)$ by a Coulomb gas technique similar to that used by Singh, Fisher and Shankar [13], expressing all the Coulomb gas quantities in terms of $\phi_{L}$ only. We find :

$$
\begin{aligned}
& \beta_{\text {bulk }}(g)=-2 \pi g^{2}+O\left(g^{3}\right), \\
& \gamma_{\text {edge }}(g)=1-2 \pi g+O\left(g^{2}\right) .
\end{aligned}
$$

The result for $\gamma_{\text {edge }}$ is different from the bulk result $\gamma(g)=1 / 2-\pi g / 2:$ the constant term is due to the edge scaling dimension of the spin operator which is twice the bulk value. The $O(g)$ coefficient leads directly to the logarithmic correction. The solution of Eq. (11) by the method of characteristics leads to an asymptotic behavior which includes a logarithmic correction :

$$
G(t)^{e d g e} \propto \frac{1}{t^{2}}(\log t)^{2} .
$$

This is the peculiar law of time decay for the autocorrelation of the end spin of the chain (in the bulk it is $\left.\log ^{1 / 2} t / t\right)$. This result holds for the staggered as well as the uniform part of the correlation function. We have checked that Eqs. (12,13) are consistent with isotropy : $\left\langle S_{0}^{+}(t) S_{0}^{-}(0)\right\rangle$ and $\left\langle S_{0}^{z}(t) S_{0}^{z}(0)\right\rangle$ have the same behavior. To treat the effect of a nonzero temperature we impose periodic conditions in Euclidean time with period $1 / T$. The system lives then on a half-cylinder. The Callan-Symanzik equation (11) is unchanged, though the Green's function depends upon an additional argument which is the temporal extent $1 / T$. A standard finite-size scaling argument leads then to a solution of the form :

$$
G(t, T)^{e d g e} \propto \frac{T^{2}}{\sinh ^{2}(\pi T t)}(\log t)^{2} .
$$

Such a behavior leads to a relaxation rate $\left(1 / T_{1}\right)^{\text {edge }} \propto$ $T \log ^{2} T$ strikingly different from the bulk. Experimental check of this result seems feasible with the possible complication that if the nearest-neighbor coupling needed in edge NMR is dipolar then it may be that there are parasitic effects due to the other neighboring chains. We note that, while we find the well-known doubling of surface exponents for the leading power law, this doubling does not extend to the power of the logarithm.

The Luttinger liquid. We now apply the same line of arguments to the one-dimensional Luttinger liquid. There are many examples of physical systems including quantum wires, carbon nanotubes or one dimensional organic conductors that belong to the universality class of the "Luttinger liquid". In such systems, the spin and charge degrees of freedom are decoupled in the low-energy longwavelength limit and can be described in a bosonization framework by two bosonic fields, $\phi_{\sigma}$ for the spin and $\phi_{\rho}$ for the charge. For isotropic systems, the effective theory for $\phi_{\sigma}$ is a free field theory like Eq. (1). The theory for $\phi_{\rho}$ is also a free theory but with a nontrivial constant $K_{\rho}$ which encapsulates all low-energy long-distance properties of the system. The rate $1 / T_{1}$ is a probe of the spin fluctuations through the spin density wave (SDW) correlations $\left\langle S D W^{z}(t) S D W^{z}(0)\right\rangle$ where the $S D W^{z}$ operator is given by $c_{n \alpha}^{\dagger} \sigma_{\alpha \beta}^{z} c_{n \beta}$ (we consider the isotropic case). In a translationally invariant system these correlations have a uniform part with $q \approx 0$ and a $q \approx 2 k_{F}$ part. They both contribute to $\left(1 / T_{1}\right)^{\text {bulk }}$ leading to a law $T+T^{K_{\rho}}$ which involves explicitly the coupling $K_{\rho}$. In the case of edge NMR, we use the bosonized form of the SDW operator :

$$
S D W_{\text {staggered }}^{z} \sim \sin \sqrt{2 \pi K_{\rho}} \phi_{\rho} \sin \sqrt{2 \pi} \phi_{\sigma} .
$$

The condition $\left\langle S^{z}\right\rangle=0$ on the last spin of the chain leads to the Dirichlet boundary conditions $\phi_{\sigma}(0)=0$ and $\phi_{\rho}(0)=\sqrt{\pi} / 2 \sqrt{2 K_{\rho}}$. The staggered part contributes then to $1 / T_{1}$ through the product :

$$
\begin{gathered}
\left\langle\sin \sqrt{2 \pi} \phi_{\sigma}(t) \sin \sqrt{2 \pi} \phi_{\sigma}(0)\right\rangle_{\phi_{\sigma}(0)=0} \\
\times\left\langle\sin \sqrt{2 \pi K_{\rho}} \phi_{\rho}(t) \sin \sqrt{2 \pi K_{\rho}} \phi_{\rho}(0)\right\rangle_{\phi_{\rho}(0)=\frac{\sqrt{\pi}}{2 \sqrt{2 K_{\rho}}}}
\end{gathered}
$$


The spin part in Eq. (17) is exactly the same as in a quantum spin chain while the charge part is different due to the presence of $K_{\rho}$ but also due to the peculiar boundary condition. As a consequence, when we compute these correlations each factor involves four nonzero contributions instead of two in the bulk (the electric neutrality of Coulomb gas calculations is affected by the boundary). Let us compute the charge correlator between two arbitrary points $\left(x_{1}, t_{1}\right)$ and $\left(x_{2}, t_{2}\right)$. We give some details in the zero temperature case, a conformal mapping can be used afterwards to obtain the corresponding expressions valid for low $T$. The left and right components of the charge Boson are related by $\phi_{\rho R}(x, t)=-\phi_{\rho L}(-x, t)+\sqrt{\pi} / 2 \sqrt{2 K_{\rho}}$ and the charge part $C_{\rho}$ of the correlator Eq. (17) is thus equal to :

$$
\begin{aligned}
& \left\langle\cos \sqrt{2 \pi K_{\rho}}\left(\phi_{\rho L}\left(x_{1}, t_{1}\right)-\phi_{\rho L}\left(-x_{1}, t_{1}\right)\right) \times\right. \\
& \left.\times \cos \sqrt{2 \pi K_{\rho}}\left(\phi_{\rho L}\left(x_{2}, t_{2}\right)-\phi_{\rho L}\left(-x_{2}, t_{2}\right)\right)\right\rangle .
\end{aligned}
$$

Evaluation of this correlation function leads to the following expression :

$$
C_{\rho}=\left(\frac{1}{x_{1} x_{2}}\right)^{K_{\rho} / 2}\left(I+\frac{1}{I}\right)
$$

where I is equal to :

$$
\left[\frac{\left(t_{1}-t_{2}\right)^{2}-\left(x_{1}+x_{2}\right)^{2}}{\left(t_{1}-t_{2}\right)^{2}-\left(x_{1}-x_{2}\right)^{2}}\right]^{K_{\rho} / 2} .
$$

When approaching the edge of the system, i.e. $x_{1}$ and $x_{1}$ are $O(a)$ where $a$ is the lattice spacing, the expression I of Eq. (20) goes to a constant and the correlator $\mathrm{C}$ becomes independent of time and the charge part in Eq. (17) does not contribute to the time decay. This is to be contrasted with the spin correlator in Eq. (17). There the boundary condition is simply $\phi_{\sigma}(0)=0$ and thus in the expression corresponding to Eq. (19), we find the difference $I-1 / I$ (with now $K_{\rho}$ replaced by 1 ) that decays in time according to a power law $\sim 1 / t^{2}$ We thus obtain a law of the form $T^{2} / \sinh ^{2} \pi T t$ at nonzero temperature leading to a "superuniversal" behavior $1 / T_{1}^{e d g e} \propto T$ independent of $K_{\rho}$.

We note that it has also been proposed recently 18 20 to use photoemission spectroscopy on the open end of a Luttinger liquid to probe the boundary effects. In this case the physical measurement is sensitive to the boundary spectral properties of the Luttinger liquid and also involves novel exponents that differ from those of the bulk.

In this Letter, we have shown how edge NMR can be used as a probe of some boundary conformal field theories. For the $\mathrm{S}=1 / 2 \mathrm{AF}$ spin chain, the relaxation rate scales like $T \log ^{2} T$ at the edge instead of $T^{0} \log ^{1 / 2} T$. For a Luttinger liquid with gapless spin and charge degrees of freedom it scales as $T$. This universal behavior is independent of the correlation exponent $K_{\rho}$ which rules all $1 \mathrm{D}$ behavior through anomalous power laws.

\section{ACKNOWLEDGMENTS}

We thank I. Affleck for a useful correspondence and F. David, J. F. Jacquinot, J. P. Renard for useful discussions

[1] M. Takigawa, N. Motoyama, H. Eisaki and S. Uchida, Phys. Rev. B55, 14129 (1997).

[2] S. Eggert and I. Affleck, Phys. Rev. Lett. 75, 934 (1995).

[3] M. Hagiwara et al., Phys. Rev. Lett. 5, 3181 (1990).

[4] J. Cardy, Nucl. Phys. B240, 514 (1984), ibid., B270, 186 (1986), ibid., B275, 200 (1986).

[5] T. Moriya, Prog. Theor. Phys. 28, 371 (1962).

[6] S. Sachdev, Phys. Rev. B50, 13006 (1994).

[7] A. W. Sandvik, Phys. Rev. B52, 9831 (1995).

[8] M. Takigawa, N. Motoyama, H. Eisaki and S. Uchida, Phys. Rev. Lett. 76, 4612 (1996).

[9] See e. g. the contribution by D. Jérome, in Organic Superconductors: from (TMTSF) ${ }_{2} P_{6}$ to Fullerenes, p. 405, M. Dekker, New York, 1994.

[10] see e.g. papers in M. Stone (ed.), Bosonization, World Scientific, Singapore, 1994.

[11] S. Eggert and I. Affleck, Phys. Rev. B46, 10866 (1992).

[12] I. Affleck, D. Gepner, H. J. Schulz and T. Ziman, J. Phys. A 22, 511 (1989).

[13] R. R. P. Singh, M. Fisher and R. Shankar, Phys. Rev. B39, 2562 (1989).

[14] S. Eggert, I. Affleck and M. Takahashi, Phys. Rev. Lett. 73, 332 (1994).

[15] N. Motoyama, H. Eisaki and S. Uchida, Phys. Rev. Lett. 76, 3212 (1996).

[16] K. Hallberg, P. Horsch and G. Martinez, Phys. Rev. B52, R719 (1995). T. Koma and N. Mizukoshi, J. Stat. Phys. 83, 661 (1996).

[17] V. Barzykhin and I. Affleck, e-print cond-mat/9810075.

[18] S. Eggert, A. Mattsson and J. M. Kinarets, Phys. Rev. B56, 15537 (1997).

[19] S. Eggert, H. Johannesson and A. Mattsson, Phys. Rev. Lett. 76, 1505 (1996).

[20] A. Mattsson, S. Eggert and H. Johannesson, Phys. Rev. B56, 15615 (1997). 